\title{
Localisation of divalent metal transporter 1 (DMT1) to the microvillus membrane of rat duodenal enterocytes in iron deficiency, but to hepatocytes in iron overload
}

\author{
D Trinder, P S Oates, C Thomas, J Sadleir, E H Morgan
}

\begin{abstract}
Background-The mechanism of iron absorption by the intestine and its transfer to the main iron storage site, the liver, is poorly understood. Recently an iron carrier was cloned and named DMT1 (divalent metal transporter 1 ).

Aims-To determine the level of DMT1 gene expression and protein distribution in duodenum and liver.

Methods-A DMT1 cRNA and antibody were produced and used in in situ hybridisation and immunohistochemistry, respectively, in rats in which the iron stores were altered by feeding diets with normal, low, and high iron content.

Results-Duodenal DMT1 mRNA was low in crypts and increased at the crypt-villus junction in iron deficient and control rats; it fell in the iron loaded state. Staining for DMT1 protein was not detected in crypts. In villus enterocytes, protein staining was localised to the microvillus membrane in iron deficiency, in the cytoplasm and to a lesser extent in the membrane in controls, and entirely in the cytoplasm of iron loaded animals. Liver DMT1 mRNA was distributed evenly across hepatocytes. DMT1 protein staining was observed on hepatocyte plasma membranes, with highest values in the iron loaded state, lower values in control animals, and none after iron depletion.
\end{abstract}

Conclusions-Results are consistent with a role for DMT1 in the transmembrane transport of non-transferrin bound iron from the intestinal lumen and from the portal blood.

(Gut 2000;46:270-276)

Keywords: DMT1; iron transporters; iron absorption; duodenum; liver

Iron is essential for the function of almost all cells due to its role in oxygen transport, energy production, and cell proliferation. Cell membranes are relatively impermeable to iron, so that its transport across these structures must be mediated by iron carriers. In the duodenum where the majority of iron is absorbed, it is proposed that at least one iron carrier exists on the apical membrane of enterocytes, and a second on the basolateral surface to transfer iron to the bloodstream. ${ }^{1}$ Once absorbed it is believed that nearly all the iron is bound by the plasma protein transferrin; it is taken up by most types of cells by transferrin receptor mediated endocytosis. This process involves the internalisation of the transferrin-receptor complex into an endosome where the iron is released from transferrin and is then transported across the endosomal membrane to the cell cytosol by an iron carrier. ${ }^{1}$ Irrespective of the way the iron is presented to the cell, either as transferrin bound iron or non-transferrin bound iron such as in the absorptive process, the mechanisms of iron transport across cell membranes are poorly understood.

Recently Gunshin et al, ${ }^{2}$ using expression cloning of mRNA derived from rat duodenum, identified a gene which encoded a protein capable of transporting metal ions, including iron, which was previously called divalent cation transporter 1 (DCT1) and has been renamed divalent metal transporter 1 (DMT1). The product of this gene is homologous to a natural resistance associated macrophage protein (Nramp1) which is associated with natural resistance to infection with intracellular parasites. ${ }^{3}$ Although DMT1 was isolated from duodenal tissue, it is likely to function as a carrier of iron in a variety of tissues. This assumption is based on the findings that high stringency northern analysis of DMT1 mRNA from different tissues showed that the gene is widely expressed and is found in the alimentary canal, bone marrow, and liver, tissues which have very important roles in iron metabolism. The strongest evidence to date for a central role of DMT1 in iron metabolism is derived from two recent studies by Fleming et $a l,{ }^{45}$ who identified by positional cloning and PCR that a missense mutation G185R in the DMT1 gene is responsible for the microcytic, hypochromic anaemia seen in mk mice and Belgrade rats. Homozygous mk mice and Belgrade rats have a severe defect in intestinal absorption and erythroid iron utilisation. ${ }^{6-9}$ This mutation in DMT1 is thought to cause distortion of the transmembrane transport channel, restricting movement of iron into the cells as well as increasing the rate of DMT1 degradation. ${ }^{10}$

In view of these findings, it is likely that DMT1 is important in the transport of iron by both transferrin receptor and non-transferrin

Abbreviations used in this paper: DCT1, divalent cation transporter 1; DMT1, divalent metal transporter 1; IRE, iron responsive element; Nramp, natural resistance associated macrophage protein. 
receptor mediated processes. In order to determine the function of DMT1 in iron metabolism it is crucial to study its tissue and cellular distribution. Therefore, we produced a specific rat DMT1 cRNA and antibody to study the level of gene expression and protein distribution along the length of the crypt-villus axis of the duodenum in rats with altered iron stores to give insight into factors that may control iron absorption and the regulation of expression of this gene. As the liver is the major storage site of excess iron absorbed from the diet it is likely that DMT1 may mediate the uptake of this iron. Therefore, we have determined the location of DMT1 in livers from animals with differing iron stores and compared this distribution with that seen in the duodenum.

\section{Methods}

ANIMALS

Twenty seven normal, weanling outbred male Wistar rats were obtained from the Animal Resource Centre (Perth, Australia). The rats were placed on a semipurified diet containing three concentrations of iron, the composition of which has been published elsewhere. ${ }^{11}$ Nine rats were fed the control diet containing a normal concentration of iron, $70 \mathrm{mg}$ iron $/ \mathrm{kg}$ diet, in the form of ferrous ammonium sulphate; nine rats were fed the iron loaded diet containing $30 \mathrm{~g}$ of carbonyl iron per kg diet; and nine rats were fed the iron deficient diet containing no added iron (iron content, $10 \mathrm{mg} / \mathrm{kg}$ ). These diets were available to the animals ad libitum for $8-10$ weeks. The animals were then killed by injection of Nembutal (Boehringer Ingelheim, Sydney, Australia) at a dose of 100 $\mathrm{mg} / \mathrm{kg}$.

PRODUCTION OF DMT1 ANTIBODY

A peptide (CVKPSQSQVLRGMFV) was synthesised according to the predicted amino acid sequence obtained from the rat DMT1 cDNA corresponding to codons 230-243 of the protein between putative transmembrane regions 5 and $6 .^{2}$ This peptide was selected because it is in an extracellular region of the protein away from the predicted glycosylation sites and with only six amino acids in common with human Nramp1. Comparison of the peptide sequence with sequence databases using the BLAST computer program ${ }^{12}$ identified only Nramp2 (DMT1) sequences. The peptide was conjugated to bovine serum albumin using activated maleimide (Pierce, Sydney, Australia). The bovine serum albumin conjugated peptide (350 $\mu \mathrm{g}$ protein) in complete Freund's adjuvant was injected subcutaneously into rabbits, and a second injection of $50 \mu \mathrm{g}$ protein in incomplete Freund's adjuvant was made four weeks later. The rabbits were bled after three weeks and the production of antibodies was confirmed by enzyme linked immunosorbent assay (ELISA). The rabbits were given a further injection of $50 \mu \mathrm{g}$ four weeks later and bled at three week intervals for determination of antibody titre.

Antibodies to bovine serum albumin were removed from the antiserum and preimmune serum using affinity chromatography. Bovine serum albumin was coupled to $\mathrm{CNBr}$ activated Sepharose 4B (Amersham Pharmacia Biotech, Melbourne, Australia) according to the manufacturer's instructions and mixed with the antiserum or preimmune serum for 16 hours at $4^{\circ} \mathrm{C}$. The resin was removed by centrifugation and the antiserum was dialysed against phosphate buffered saline (PBS) $\mathrm{pH}$ 7.4. The removal of bovine serum albumin antibody was confirmed by ELISA.

DMT1 CLONE AND SUBCLONING

Mucosal tissue from an iron deficient rat was obtained from the mid villus region of the duodenum by differential scrapings as follows. Initially, the apical mucosa was gently scaped with a glass microscope slide and the separated tissue was discarded. Then a second stronger scrape was applied and about $100 \mathrm{mg}$ of mucosal tissue was obtained and used for RNA purification using RNAzol B (Tel-Test, Inc., Texas, USA). From the published sequence of the rat DMT1 gene (Genbank accession number af 008439) a set of forward and reverse primers were synthesised: 5'-TTTTTGGCTTTCTCATCACTATCATGGCCC-3' and 5'ATTGGCTTCTCGAACTTCCTGCTTATTGGC-3', respectively. These sequences encode transmembrane domains 6 and 7 of the protein and are common to both the iron responsive element (IRE) form and non-IRE form of DMT $1 .{ }^{25}$ Using these primers, $1 \mu \mathrm{g}$ of total RNA, and an Access RT-PCR kit (Promega, Sydeny, Australia), a fragment was amplified. It had the predicted size of $252 \mathrm{bp}$. This product was purified and subcloned into pGEM-T Easy Vector (Promega, Sydney, Australia). The identity of the insert was confirmed by sequencing to correspond to 731-983 bp of the DMT1 gene. Following transformation, amplification, and purification, inserts were identified by restriction enzyme digestion. The DMT1 template was linearised with either Pst1 or Sph1. Sense and antisense riboprobes were synthesised using SP6 and T7 polymerase, respectively, and ${ }^{35} \mathrm{~S}$-labelled uridine 5 ' triphosphate (Amersham Pharmacia Biotech, Melbourne Australia) as described recently. ${ }^{11}$ The antisense riboprobe will recognise both IRE and non-IRE forms of DMT $1 .^{25}$

\section{MORPHOLOGICAL STUDIES}

After deep anaesthesia was established, a midline incision was made in the rats to expose the thorax and abdomen. Segments of the duodenum and liver were removed and immediately placed into buffered formol saline ( $\mathrm{pH}$ 7.4). The tissues were subsequently processed for embedding in paraffin wax. Sections $(5 \mu \mathrm{m})$ were cut, floated onto slides coated with either 3-aminopropylethoxysilane (in situ hybridisation) or $0.5 \%$ gelatine (immunohistochemistry), and allowed to dry. The sections were then dewaxed, rehydrated in PBS ( $\mathrm{pH}$ 7.4), and used in the procedures described below. Immunological studies were also performed on hepatocytes isolated from normal rat liver as described previously, ${ }^{13}$ fixed in $10 \%$ buffered 

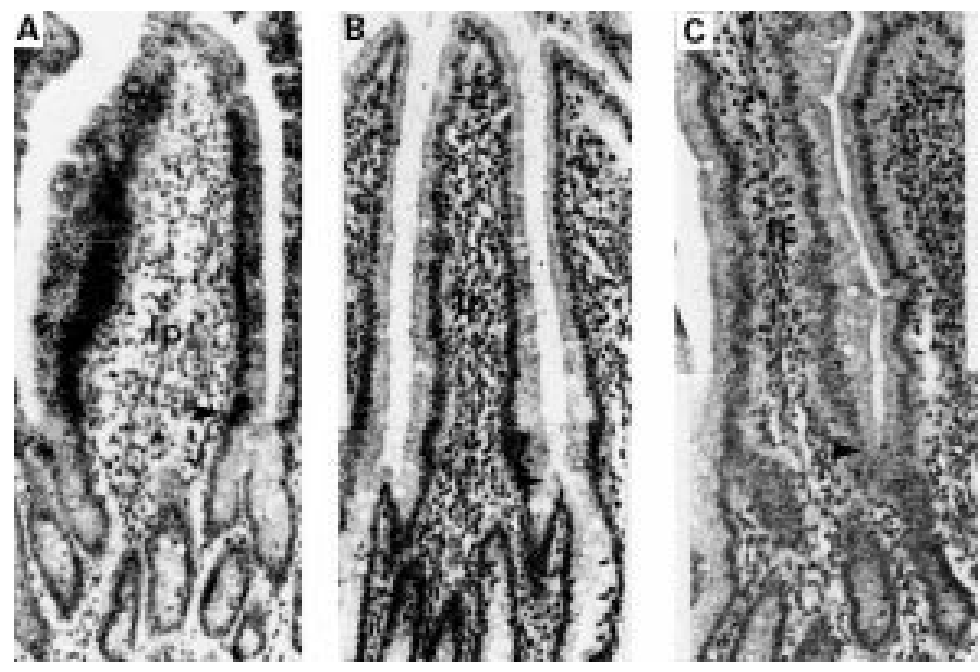

Figure 1 In situ hybridisation using an antisense rat DMT1 cRNA of the duodenum of an iron deficient $(A)$, control $(B)$, and iron loaded $(C)$ rat. In all cases low levels of DMT1 $m R N A$ expression were seen in cells lining the upper half of the crypt (below arrow). The signal intensity was least with iron deficiency and greatest with iron loading. DMT1 $m R N A$ increased greatly at the crypt-villus junction (arrow) in controls and to a much greater extent in iron deficient tissue. DMT1 gene expression was absent in the enterocytes of the upper quarter of the villus (above arrow) in all iron states. No expression was seen in lamina propria cells (lp). Original magnification $\times 150$.

formol saline, cytocentrifuged, and then reacted with DMT1 antibody as described below.

\section{IN SITU HYBRIDISATION}

This procedure was performed on at least six sections of duodenum and liver from five iron deficient, control, and iron loaded animals using methods described elsewhere. ${ }^{11}$ One million dpm of either sense or antisense riboprobe were applied to each tissue in a volume of $25 \mu \mathrm{l}$ and the tissues were exposed to LM1 photographic emulsion (Amersham Pharmacia Biotech, Melbourne, Australia) for five days before development. The sections were stained with haematoxylin and eosin.

IMMUNOHISTOCHEMICAL LOCALISATION OF DMT1 Immunohistochemistry was performed on at least six sections of duodenum and liver from five iron deficient, control, and iron loaded animals. To eliminate endogenous peroxidase, rehydrated tissue was reacted with $2.5 \%$ aqueous periodic acid for five minutes and $0.02 \%$ sodium borohydride for two minutes. The tissues were washed with PBS containing saponin $(0.2 \%)$ and incubated with PBSsaponin containing rabbit antirat DMT1 antibody $(1 / 500)$ overnight at $4^{\circ} \mathrm{C}$. The tissues were washed three times with PBS-saponin and incubated with a sheep antirabbit IgG peroxidase coupled secondary antibody (1/100; Silenus, Amrad Biotech, Melbourne, Australia) for two hours at room temperature, followed by three washes with PBS-saponin, and one with $0.05 \mathrm{M}$ Tris ( $\mathrm{pH}$ 7.4). Localisation of the protein was revealed by reaction with $0.05 \%$ 3',3'diaminobenzidine tetrahydrochloride and $0.01 \%$ hydrogen peroxide. The tissue was dehydrated and mounted in DePeX mounting medium (BDH, Melbourne, Australia). Sections used for control staining were treated identically except that preimmune rabbit serum was substituted for DMT1 antiserum.
Specificity of the antibody was determined by incubating sections of duodenum and liver from rats of varying iron status with DMT1 antibody (1/500) in the presence of $50 \mu \mathrm{g}$ DMT1 peptide before processing; at the same time, other sections were incubated with the antibody in the absence of the peptide.

\section{Results}

The antiserum used in these studies was raised against a unique peptide with amino acid sequence predicted from the cDNA sequence of rat DMT1, conjugated to bovine serum albumin. After the antibodies against albumin were removed the antiserum showed on western blot analysis of duodenal mucosa scraping from an iron deficient rats a single band with an apparent molecular weight of 97 $\mathrm{kDa}$. Gruenheid et al have shown that DMT1 has a similar apparent molecular weight $(90-100 \mathrm{kDa})$. This was higher than that calculated from the predicted amino acid sequence of rat DMT1 $(62 \mathrm{kDa})$ due to post-translational glycosylation of the protein. ${ }^{14}$ The specificity of the antibody against DMT1 peptide was shown by the elimination of immunohistochemical staining in the duodenum (fig 2A, C) and liver (fig 3D, E) by addition of peptide to the antiserum during incubation of tissue sections. These studies indicate that the antiserum is monospecific and reacts against a 14 amino acid peptide derived from rat DMT1. Hence, there can be little or no doubt that it is an antibody against rat DMT1. Moreover, the tissue distribution and relative intensity of staining with the antibody was similar to that described for DMT1 $\mathrm{mRNA}^{2}$ : high in the duodenum, kidney, and bone marrow and lower in liver, colon, and pancreas (Trinder D et al. Invited Symposium at 5 th Internet World Congress on Biochemical Science, at URL http://www.mcmaster.ca/ inabis98/).

The iron status of the animals was determined by measuring the hepatic non-haem iron concentrations. ${ }^{15}$ The hepatic non-haem iron concentration of animals fed an iron deficient diet was $10 \mu \mathrm{g} / \mathrm{g}$ liver wet weight while the hepatic non-haem iron concentrations in the control and iron loaded animals were 15- and 220 -fold greater than iron deficient concentrations, respectively.

In situ hybridisation using an antisense rat DMT1 riboprobe revealed low levels of DMT1 mRNA expression in the crypt region of the duodenum of the iron deficient (fig 1A), control (fig 1B), and iron loaded (fig 1C) tissues. Immunohistochemical staining of DMT1 protein revealed there was no detectable expression of DMT1 protein in the crypt epithelial cells from iron deficient (fig 2A), control (fig 2D), and iron loaded tissues. As the morphological appearances of control and iron loaded duodenum were similar, for reasons of brevity only photomicrographs of the control tissue have been included.

In villus enterocytes DMT1 mRNA levels increased dramatically at the crypt-villus junction in the control tissue (fig 1B), and to a much greater extent in iron deficient tissue (fig 
1A), but did not increase, and may have fallen in iron loaded tissue (fig 1C). In all cases DMT1 mRNA expression decreased towards the tips of the villi and was not detectable in the lamina propria cells of either the crypts or villi (fig $1 \mathrm{~A}-\mathrm{C}$ ). Using control DMT1 sense riboprobes no signal was observed.

DMT1 protein staining was seen in enterocytes, commencing at the crypt-villus junction and increasing in intensity to reach highest levels in the upper half of the villus (fig $2 \mathrm{~A}, \mathrm{~B}, \mathrm{D}$, E). DMT1 staining was specific for enterocytes, as adjacent goblet cells did not stain for the protein. No staining of DMT1 was seen in the lamina propria, including macrophages of the duodenum from control (fig 2E), iron deficient (fig 2B), and iron loaded animals. The absence of staining in the macrophages showed that the antiserum did not cross react with Nramp1, a closely related protein expressed in macrophages. The positive staining observed using the antibody in the iron deficient duodenum (fig $2 \mathrm{~A}, \mathrm{~B}$ ) was blocked by the addition of
DMT1 peptide (fig 2C) and no staining was seen with the preimmune serum (fig $2 \mathrm{~F}$ ).

The intracellular distribution of DMT1 varied greatly in the villus enterocytes in relation to iron status (fig 2). In the duodenum of rats fed the control diet, DMT1 staining was localised mainly intracellularly with an even distribution throughout the cytoplasm, but there was also a low level of margination of the protein along the apical membrane (fig $2 \mathrm{D}, \mathrm{E}$ ). In enterocytes of the iron deficient duodenum DMT1 protein was predominantly associated with the apical membrane, with only low levels of protein staining in the cytoplasm (fig $2 \mathrm{~A}, \mathrm{~B}$ ).

In situ hybridisation was performed on liver sections from iron deficient, control, and iron loaded rats. Using a DMT1 antisense probe there was a low intensity signal distributed evenly across the hepatocytes of the liver (fig $3 \mathrm{~A})$ and there were no obvious differences in the level of expression of DMT1 mRNA with varying liver iron status. No signal was detected using a control DMT1 sense riboprobe.

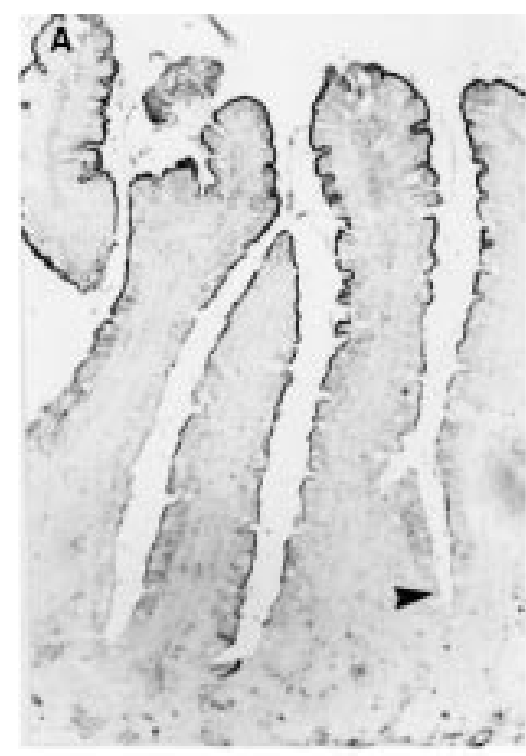

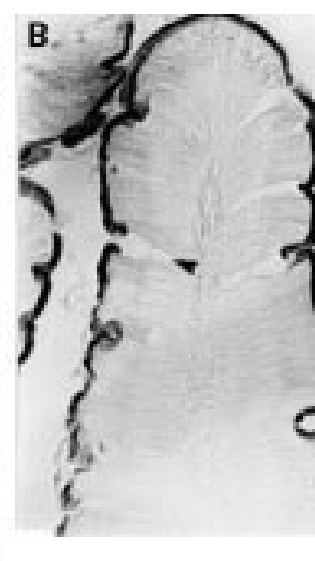

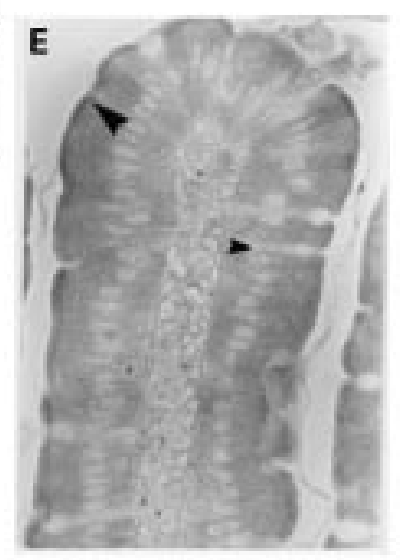

Figure 2 Immunohistochemical localisation of rat DMT1 protein using a polyclonal antibody in the duodenum of iron deficient $(A, B, C)$ and control tissue $(D, E)$. No staining was observed in epithelial cells of the crypt region (A,D; below arrow). In the villus region (above arrow) DMT1 staining commenced at the crypt-villus junction (arrow) and increased in intensity to reach highest levels in enterocytes of the upper half of the villus tip. Goblet cells did not stain (B,E; small arrow). DMT1 in control enterocytes (E) was localised intracellularly with an approximately even distribution throughout the cytoplasm. The nuclei were negative. Some margination of the DMT1 protein along the apical membrane could be observed (E; large arrow). In enterocytes of the iron deficient duodenum DMT1 was predominantly associated with the apical membrane $(A, B)$. Low concentrations of protein could also be seen intracellularly in the apical cytoplasm $(A, B)$. No staining was seen in the iron deficient duodenum using either DMT1 antibody in the presence of the DMT1 peptide $(C)$ or preimmune serum $(F)$. Original magnification $\times 85(A, D), \times 225(B, E, F), \times 60(C)$. 
DMT1 protein staining was not detected in livers from the iron deficient animals (fig 3B). In contrast, DMT1 staining was associated with the cells along the lining membrane of the sinusoids in control animals (fig 3C). The distribution of staining for DMT1 was consistent with the presence of the protein on the microvillous membrane of hepatocytes, but it could not be excluded that staining of endothelial cells also occurred. No staining of Kupffer cells was observed. A similar distribution of the protein staining was seen in the iron loaded livers but the intensity of staining was much greater (fig 3D) than in the control livers. The positive
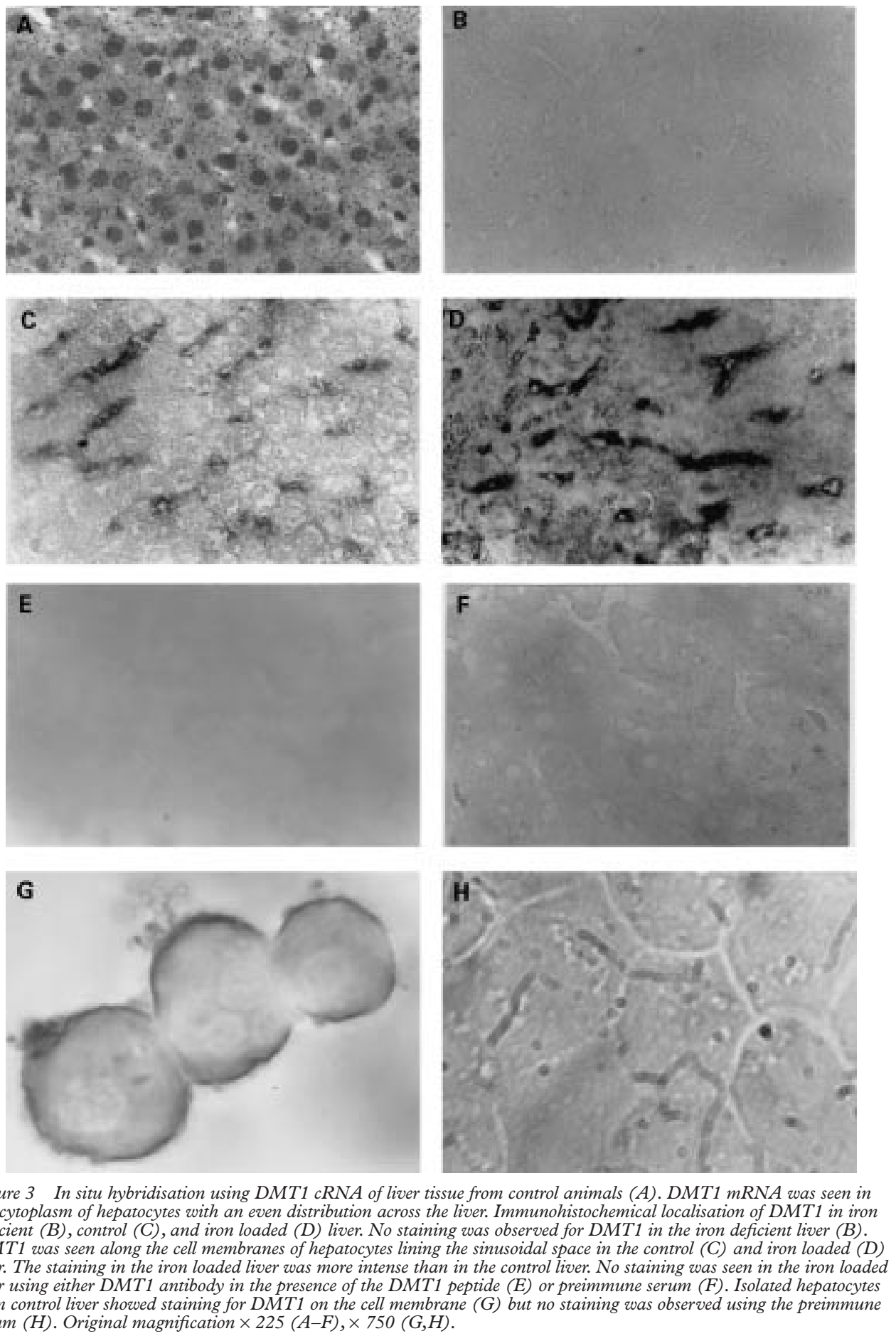

Figure 3 In situ hybridisation using DMT1 cRNA of liver tissue from control animals (A). DMT1 mRNA was seen in the cytoplasm of hepatocytes with an even distribution across the liver. Immunohistochemical localisation of DMT1 in iron deficient (B), control (C), and iron loaded (D) liver. No staining was observed for DMT1 in the iron deficient liver (B). $D M T 1$ was seen along the cell membranes of hepatocytes lining the sinusoidal space in the control (C) and iron loaded (D) liver. The staining in the iron loaded liver was more intense than in the control liver. No staining was seen in the iron loaded liver using either DMT1 antibody in the presence of the DMT1 peptide $(E)$ or preimmune serum $(F)$. Isolated hepatocytes from control liver showed staining for DMT1 on the cell membrane $(G)$ but no staining was observed using the preimmune serum $(H)$. Original magnification $\times 225(A-F), \times 750(G, H)$.

staining seen with the antibody in the iron loaded livers (fig 3D) was blocked in the presence of DMT1 peptide (fig 3E) and no staining was seen with the preimmune serum (fig $3 F$ ).

In order to determine whether the DMT1 staining observed in the sinusoidal region was present in hepatocytes, studies were performed using isolated hepatocytes from control livers. DMT1 was found on these cells, associated only with the cell membranes; the cytoplasm was not stained (fig 3G). DMT1 protein staining was not confined to the microvillus membrane of the hepatocytes, suggesting the cells had lost their polarity during the isolation
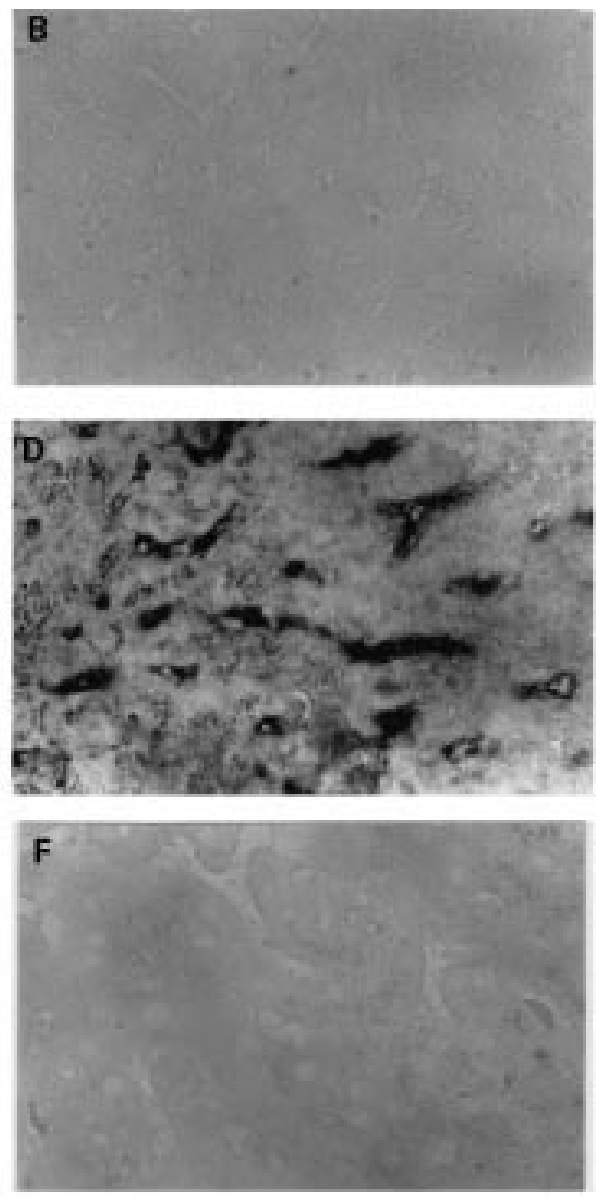
procedure. No staining was seen using preimmune serum (fig 3H). The presence of DMT1 staining on isolated hepatocytes supports our findings in whole liver sections that the DMT1 seen on the sinusoidal membrane was associated with hepatocytes.

\section{Discussion}

In the crypt epithelium of the duodenum low levels of DMT1 mRNA were detected in all three conditions of iron status. However, no DMT1 protein was detected, suggesting that either the gene was not translated or that the concentrations of the protein produced are too low to be detected by immunohistochemistry. At the crypt-villus junction, there was a notable increase in DMT1 mRNA compared with crypt cells in iron deficient animals and to a lesser extent in control animals. By contrast, the mRNA level did not increase in iron loaded rats. This change in DMT1 gene expression at the crypt-villus junction occurs at the same time as the enterocyte matures and acquires the proteins needed for digestion and absorption. Coinciding with this, DMT1 protein was first detected at the crypt-villus junction and reached highest concentrations in the upper half of the villus. It is recognised that in situ hybridisation provides only a semiquantitative measure of mRNA levels, especially when comparing one section with another. However, the sections in fig 1 extend from the crypt to the villus region and they each provide an internal control over the conditions used during in situ hybridisation, and allow for a greater degree of confidence in relation to relative mRNA levels between villus and crypt. This is clearly highest in iron deficiency and least in iron overload. These results suggest that a change in the regulation of DMT1 mRNA gene expression occurs as enterocytes migrate from the crypt to the villus regions of the duodenal mucosa. The results in the villus region are compatible with post-transcriptional regulation by cellular iron concentrations in a manner similar to that described for the transferrin receptor ${ }^{16}$ : in iron deficiency a cytosolic iron regulatory protein binds to the receptor mRNA at an IRE, increasing its stability and translation of the message. As DMT1 mRNA may contain an IRE, ${ }^{2}$ a similar mechanism could operate in the duodenal villi.

The above hypothesis seems unlikely to apply to the liver, since DMT1 protein staining was greatest in iron loading and least in iron deficiency. The regulatory mechanism here is uncertain, but it may be related to the identification of an alterative splicing site at the $\mathrm{C}$ terminal exon of DMT1 so that DMT1 can be expressed with or without the putative IRE. ${ }^{17}$ Hence, it is possible that in the liver the major form of DMT1 mRNA that is expressed is the one deficient in an IRE, and that its expression is regulated in a positive way by iron concentrations. The observation that the DMT1 gene contains metal responsive elements in its 5' regulatory region ${ }^{17}$ may provide an explanation for this.

In villus epithelial cells of control and iron loaded animals DMT1 protein staining was found largely at intracellular sites and specifically in enterocytes (goblet cells were negative); in enterocytes from iron deficient animals the protein was located predominantly on the luminal membrane. This suggests that DMT1 may shuttle back and forth from the cytoplasm to the cell membrane. If this is the case it is reminiscent of other transport systems-for example, the GLUT-4 glucose transporter stimulated by insulin ${ }^{18}$ and aqueporin 2 involved in water reabsorption in the presence of vasopressin in the distal nephron. ${ }^{19}$ In the absence of ligands both transporters are found in the membrane of intracellular vesicles. However, on ligand binding and downstream activation of intracellular signalling the vesicles migrate and fuse with the cell membrane, enabling the transport of the respective substrates. In hepatocytes DMT1 protein staining was also observed on the microvillus membrane. Whether this is a permanent localisation or occurs as part of a shuttle process as postulated for enterocytes but with lower intracellular concentrations which could not be detected by the immunohistochemical procedure, remains to be determined.

Previously it has been shown that the transmembrane transport of iron by DMT1 increases as the $\mathrm{pH}$ is reduced below neutrality. ${ }^{2}$ The low $\mathrm{pH}$ of the mucosal surface of the upper small intestine ${ }^{20}$ and the endocytic vesicles ${ }^{1}$ would provide a suitable environment for the operation of DMT1 for the absorption of iron from the diet and the transport of transferrin bound iron across the endosomal membrane after transferrin receptor mediated endocytosis. Localisation of DMT1 protein to the sinusoidal plasma membrane of hepatocytes suggests that it also plays a role in iron transport across this membrane. A low $\mathrm{pH}$ microenvironment at the surface would be expected to facilitate the transport of iron across the membrane by DMT1 but whether this occurs remains to be determined.

The effects of dietary iron status on DMT1 expression and distribution in enterocytes and hepatocytes help to explain a number of physiological observations. During absorption from the intestine iron must pass through both the microvillus and basolateral membranes of the enterocyte and it is likely that iron transporters are involved at both of these sites. ${ }^{1}$ The present results indicate that DMT1 is the transporter at the microvillus membrane but not at the basolateral membrane and also provide an explanation for why the uptake step in iron absorption (across the microvillus membrane) is enhanced in iron deficiency, and inhibited in dietary iron overload and in the homozygous Belgrade rats. ${ }^{9}$ Iron deficiency also leads to enhanced iron transport across the basolateral membrane but the mechanism involved remains to be elucidated. Other stimulators of the uptake step in iron absorption are hypoxia and anaemia, and these effects seem to be independent of iron concentrations. ${ }^{21-23}$ The promoter region of DMT1 contains two potential Hif-1 binding sites which, by analogy with erythropoietin, ${ }^{1724}$ could provide a mech- 
anism for upregulation of DMT1 in response to hypoxia.

It is generally assumed that iron absorbed from the intestine is rapidly bound by the transferrin present in portal blood and is distributed to tissues in accordance with their content of transferrin receptors. However, when the transferrin of peripheral blood is saturated or nearly saturated with iron due to injection of iron, ${ }^{25}{ }^{26}$ dietary iron overload as in genetichaemochromatosis, ${ }^{27}$ orhypotransferrinaemia, ${ }^{28}{ }^{29}$ a large proportion of iron absorbed from the intestine is cleared from the portal blood by the liver. The presence of DMT1 on the microvillus membrane of hepatocytes, increasing under conditions of iron overload, provides a logical explanation for these findings. Possibly, even under normal conditions, some of the iron absorbed from the intestine during periods of rapid absorption is not bound by transferrin by the time the portal blood reaches the liver and is cleared from the blood by the action of hepatocyte microvillus DMT1. Thus, in conditions with low plasma unsaturated binding capacity of transferrin and even normal conditions, the liver functions to clear the portal blood of non-transferrin bound iron and reduces the risk of unregulated iron uptake and damage to cells elsewhere in the body. Conversely, in iron deficiency downregulation of DMT1 expression in the liver (associated with its upregulation in the intestinal enterocyte) would see the iron in portal blood bypassing the liver and having more opportunity to bind to transferrin. It would then be distributed throughout the body in a manner regulated by the expression and function of transferrin receptors.

This work was supported by grants from the National Health and Medical Research Council of Australia and a Raine Medical Research Foundation Fellowship to DT. The authors would like to thank Alan Light for his technical assistance.

1 Morgan EH. Iron metabolism and transport. In: Zakin D and Boyer TD, eds. Hepatology: a textbook of liver disease. Philadelphia: Saunders, 1996:526-54.

2 Gunshin H, Mackenzie B, Berger UV, et al. Cloning and characterization of a mammalian proton-coupled metal-ion characterization of a mammalian proto

3 Vidal SM, Malio D, Vogan K, et al. Natural resistance to infection with intracellular parasites: isolation of a candiinfection with intracellular parasites:
date for Bcg. Cell 1993;73:469-85.

4 Fleming MD, Trenor CC, Su MA, et al. Microcytic anaemia mice have a mutation in Nramp2, a candidate iron transporter gene. Nat Genet 1997;16:383-6.

5 Fleming MD, Romano MA, Su MA, et al. Nramp2 is mutated in the anemic Belgrade (b) rat: evidence of a role for DMT1 in endosomal iron transport. Proc Natl Acad Sci USA 1998;5:1148-53.
6 Edwards JA, Hoke JE. Defects of intestinal mucosal iron uptake in mice with hereditary microcytic anemia. Proc Soc Exp Biol Med 1972;141:81-4.

7 Edwards JA, Hoke JE. Red cell iron uptake in hereditary microcytic anemia. Blood 1975;46:381-8.

8 Morgan EH, Bowen BJ. Anemia of the Belgrade rat: evidence for defective membrane transport of iron. Blood 1986;70:38-44.

9 Oates PS, Morgan EH. Defective iron uptake by the duodenum of Belgrade rats fed diets of different iron contents $\mathrm{Am}$ f Physiol 1996;270:G5826-32.

10 Su MA, Trenor CC, Fleming JC, et al. The G185R mutation distrupts function of the iron transporter Nramp2. Blood 1998;92:2157-63.

11 Oates PS, Morgan EH. Ferritin gene expression and transferrin receptor activity in intestine of rats with varying iron stores. Am f Physiol 1997;273:G636-46.

12 Altschul SF, Bogusk MS, Gish W, et al. Issues in searching molecular sequence databases. Nat Genet 1994;6:119-29.

13 Baker E, Baker SM, Morgan EH. Characterisation of non-transferrin-bound iron (ferric citrate) uptake by rat hepatocytes in culture. Biochim Biophys Acta 1998;1380: 21-30.

14 Gruenheid S, Canonne-Hergaux F, Gauthier S, et al. The iron transport protein NRMAP2 is an integral membrane glycoprotein that colocalizes with transferrin in recycling glycoprotein that colocalizes with transfe
endosomes. F Exp Med 1999;189:831-41.

15 Kaldor I. Studies on intermediary iron metabolism.V. The measurement of non-haemoglobin tissue iron. Aust f Exp Biol Sci 1954;32:780-95.

16 Klausner RD, Rouault TA, Harford JB. Regulating the fate of mRNA: the control of cellular iron metabolism. Cell 1993;72:19-28.

17 Lee PL, Gelbart T, West C, et al. The human Nramp2 gene: characterization of the gene structure, alternative splicing, promoter region and polymorphisms. Blood Cells $\mathrm{Mol}$ Dis 1998:24;199-215.

18 James DE, Piper RC, Slot JW. Insulin stimulation of GLUT-4 translocation: a model for regulated recycling. Trends Cell Biol 1994;4:120-6.

19 Nielsen S, Chou CL, Marples D, et al. Vasopressin increases water permeability of kidney duct by inducing translocation of aquaporin-CD water channels to plasma memtion of aquaporin-CD water channels to plasma
brane. Proc Natl Acad Sci USA 1995;92:1013-17.

20 McEwan GTA, Daniel D, Fett C, et al. The effect of Escherichia coli Sta enterotoxin and other sectretagogues on mucosal surface $\mathrm{pH}$ of rat small intestine in vivo. Proc $R$ Soc Lond B 1988;234:219-37.

21 Raja KB, Simpson RJ, Pippard MJ, et al. In vivo studies on the relationship between intestinal iron $\left(\mathrm{Fe}^{3+}\right)$ absorption, hypoxia and erythropoiesis in the mouse. Br $\mathcal{F}$ Haematol 1988;68:373-8.

22 Peters TJ, Raja KB, Simpson RJ, et al. Mechanisms and regulation of intestinal iron absorption. Ann NY Acad Sci 1988;256:141-7.

23 Hughes RT, Smith T, Hesp R, et al. Regulation of iron absorption in iron loaded subjects with end stage renal disease: effects of treatment with recombinant human erythropoietin of reduction of iron stores. $\mathrm{Br} \mathcal{F}$ Haematol 1992;82:445-54.

24 Semenza GL, Wang GL. A nuclear factor induced by hypoxia via de novo protein synthesis binds to human erythropoietin gene enchancer at a site required for transcriptional activation. Mol Cell Biol 1992;12:5447-54.

25 Wheby MS, Jones LG. Role of transferrin in iron absorption. f Clin Invest 1963;42:1007-16.

26 Wheby MS, Jones LG. Role of transferrin saturation on iron absorption in man. N Engl F Med 1964;271:1391-5.

27 Fawwaz RA, Winchell HS, Pollycove M, et al. Hepatic iron deposition in humans. Blood 1967;30:417-24.

28 Bernstein SE. Hereditary hypotransferrinemia with hemosidererosis, a murine disorder resembling human atransferrinemia. F Lab Clin Med 1987;110:690-705.

29 Simpson RJ, Lombard M, Raja KB, et al. Iron absorption by hypotransferrinaemic mice. Br f Haematol 1991;78:565- 